\title{
A Game Theoretic Approach to Minimize the Completion Time of Network Coded Cooperative Data Exchange
}

\author{
Ahmed Douik $^{\dagger}$, Sameh Sorour*, Hamidou Tembine ${ }^{\dagger}$, Mohamed-Slim Alouini ${ }^{\dagger}$, and Tareq Y. Al-Naffouri ${ }^{\dagger *}$ \\ ${ }^{\dagger}$ King Abdullah University of Science and Technology (KAUST), Kingdom of Saudi Arabia \\ ${ }^{*}$ King Fahd University of Petroleum and Minerals (KFUPM), Kingdom of Saudi Arabia \\ Email: ${ }^{\dagger}\{$ ahmed.douik,hamidou.tembine,slim.alouini,tareq.alnaffouri $\} @$ kaust.edu.sa \\ * $\{$ samehsorour,naffouri $\} @$ kfupm.edu.sa
}

\begin{abstract}
In this paper, we introduce a game theoretic framework for studying the problem of minimizing the completion time of instantly decodable network coding (IDNC) for cooperative data exchange (CDE) in decentralized wireless network. In this configuration, clients cooperate with each other to recover the erased packets without a central controller. Game theory is employed herein as a tool for improving the distributed solution by overcoming the need for a central controller or additional signaling in the system. We model the session by self-interested players in a non-cooperative potential game. The utility function is designed such that increasing individual payoff results in a collective behavior achieving both a desirable system performance in a shared network environment and the Pareto optimal solution. Through extensive simulations, our approach is compared to the best performance that could be found in the conventional point-to-multipoint (PMP) recovery process. Numerical results show that our formulation largely outperforms the conventional PMP scheme in most practical situations and achieves a lower delay.

Index Terms-Cooperative data exchange, instantly decodable network coding, non-cooperative games, potential game, Nash equilibrium.
\end{abstract}

\section{INTRODUCTION}

\section{A. Network Coding}

Since its introduction in [1], NC was shown to be a promising technique to significantly improve the throughput and delays of packet recovery especially in wireless erasure networks, due to the broadcast nature of their transmissions. These merits are essential for real time applications requiring reliable transmissions and fast recovery over erasure channels, such as multimedia streaming [2].

Two important classes of $\mathrm{NC}$ for such applications can be distinguished in the literature: the Random Network Coding (RNC) [3], [4] and the Opportunistic Network Coding (ONC) [5], [6]. RNC is implemented by combining packets with independent, random and non zero coefficients [3]. Despite its attractive benefits such as optimality in number of transmissions for broadcast applications and ability to recover even without feedback [4], RNC is not suitable for the applications of our interest since it does not allow progressive decoding of the frame, is not optimal for multipoint to multipoint communications (multicast) and require expensive computation at the clients to decode the frame. In ONC, packet combinations are selected according to the received/lost packet state of each client [6]. ONC was show to be a graceful solution for packet recovery for wireless network [7].

One ONC subclass that suits most of the aforementioned application is the instantly decodable network coding (IDNC) since it provides instant and progressive decoding of packets. IDNC can be implemented using binary XOR to encode and decode packets. Furthermore, no buffer is needed at the clients to store non instantly decodable packets for future decoding possibilities. Thanks to its merits, IDNC was an intensive subject of research [2], [8]-[20]. In [21], the authors studied the problem of minimizing the completion time in IDNC. The completion time was proofed to be related to the decoding delay in [22] and can be better controlled with it.

\section{B. Motivation and Related Work}

In all aforementioned works, the base station of a point-tomultipoint network (such as cellular, Wi-Fi and WiMAX and roadside to vehicle networks) was assumed to be responsible for the recovery of erased packets. This can pose a threat on the resources of such base stations and their abilities to deliver the required huge data rates especially in future wireless standards. This problem becomes more severe in roadside to vehicle networks since the vehicles usually bypass the roadside senders very fast and thus cannot rely on it for packet recovery but rather on completing the missing packets among themselves. One alternative to this problem is the notion of cooperative data exchange (CDE) introduced in [23]. In this configuration, clients can cooperate to exchange data by sending IDNC recovery packets to each other over short range and more reliable communication channels, thus allowing the base station to serve other clients. This CDE model is also important for fast and reliable data communications over ad-hoc networks, such vehicular and sensor networks. Consequently, it is very important to study the minimization of delays and number of transmissions in such IDNC-based CDE systems.

Unlike conventional point-to-multipoint scenario, the IDNC based CDE systems require not only decisions on packet 
combinations but also on which client to send in every transmission in order to achieve a certain quality for one of the network metrics. Recently Aboutorab and al. [20] considered the problem of minimizing the sum decoding delay for CDE in a centralized fashion. By centralized, we mean that a central unit (such as the base station in the cellular example) takes the decisions on which client to send which packet combination in each transmission.

\section{Contributions}

In this paper, we introduce a game theoretic framework for studying the problem of minimizing the completion time of instantly decodable network coding for cooperative data exchange in decentralized wireless network. The problem is modeled as cooperative control problem using game theory as a tool for improving the distributed solution by overcoming the need for a central controller or additional signaling in the system.

Cooperative control problems entail numerous autonomous players seeking to collectively achieve a global objective. The network coding problem is one example of a cooperative control problems, in which the global objective is for all players to efficiently use a common resource by opportunistically taking advantage of the possible coding occasions. The central challenge in cooperative control problems is to derive a local control mechanism for the individual players such that the players operate in a manner that collectively serves the desired global objective.

In this paper, we derive expressions for the individual utility functions in such way that increasing individual payoff results in a collective behavior achieving a desirable system performance in a shared network environment. We then improve the game formulation to include punishment policy and reduce the set of equilibrium to the one dimensional line containing the Pareto optimal solution of our interest. To the best of our knowledge, using game theory tools to model IDNC-based $\mathrm{CDE}$ has not been addressed in the literature and only heuristic algorithm were proposed to solve the problem in a centralized fashion [20]. Moreover, this work can serve as a background to build more complicated system in which the feedback or the players state are not available.

The rest of this paper is divided as follows: Background about game theory and specially potential games is briefly recalled in Section III In Section III. we present our network model and protocol. The game parameters, and formulation are presented in Section IV The punishment policy and the new game formulation are provided in Section V. In Section VI. we present the game equilibrium and the algorithm used to simulate the system. Before concluding in Section VIII. simulations results are illustrated in Section VII.

\section{BACKGROUND: NON-COOPERATIVE GAMES}

\section{A. Definitions and Notations}

We define a finite stochastic non-cooperative game, like in [24], with a common state by the 6-uplet:

$$
\mathcal{G}=\left(\mathcal{M},\left\{\overline{\mathcal{A}}_{i}\right\}_{i}, \Omega,\left\{\mathcal{A}_{i}\right\}_{i},\left\{\alpha_{i}\right\}_{i}, q,\left\{\mathcal{U}_{i}\right\}_{i}\right),
$$

where:

- $\mathcal{M}=\{1, \ldots, M\}$ is the set of players,

- $\left\{\overline{\mathcal{A}}_{i}\right\}_{i}$ is the set of all possible actions during the course of the game,

- $\Omega$ is the set of possible states of the game,

- $\mathcal{A}_{i}(\omega)$ is the set of possible action for player $i$ in the state $w \in \Omega$ of the game,

- $\alpha_{i}: \Omega \longrightarrow 2^{\overline{\mathcal{A}}_{i}}$ is the correspondence determining the possible actions at a given state of a game,

- $q_{t}$ is the conditional distribution of the transition probability from state to state. For independent states games, $q$ is the distribution over the set $\Omega$ and can be ignored in the definition of the game. Otherwise the game is called competitive Markov decision process [25],

- $\mathcal{U}_{i}$ is the utility function of player $i$, which will be defined further in the paper.

Let $\omega(t) \in \Omega$ be the state of the game at the stage $t$. For notation simplicity, the set of actions of player $i$ at stage $t$ will be denoted by $\mathcal{A}_{i}(t)$ instead of $\mathcal{A}_{i}(\omega(t))$. Let $\mathcal{A}(t)=$ $\mathcal{A}_{1}(t) \times \ldots \times \mathcal{A}_{M}(t)$ be the set of all possible actions that can be taken by all the players at the stage $t$ of the game.

For an action profile $\underline{a}_{t}=\left(a_{1}(t), \ldots, a_{M}(t)\right)^{\mathbf{T}} \in \mathcal{A}(t)$, let $\underline{a}_{t,-i}$ denote the profile of players other than player $i$. In other words, $\underline{a}_{t,-i}=\left(a_{1}(t), \ldots, a_{i-1}(t), a_{i+1}(t), \ldots a_{M}(t)\right)^{\mathbf{T}}$. The subscript ${ }^{\mathbf{T}}$ denote the transpose operator. We can write a profile $\underline{a}_{t}$ of actions as $\left(\underline{a}_{t, i}, \underline{a}_{t,-i}\right)$. Similarly, the notation $\mathcal{A}_{-i}(t)=\prod_{j \neq i} \mathcal{A}_{j}(t)$ refers to the set of possible actions of all the player other than player $i$ at stage $t$ of the game. Let $\underline{h}_{t}=(\boldsymbol{\omega}(1), \underline{a}(1), \ldots, \underline{a}(t-1), \boldsymbol{\omega}(t))^{\mathbf{T}}$ be the history of the game at stage $t$ that lies in the set:

$$
\mathcal{H}_{t}=\left(\bigotimes_{t^{\prime}=1}^{t-1} \Omega \times \mathcal{A}\left(t^{\prime}\right)\right) \times \Omega .
$$

The utility function $\mathcal{U}_{i}$ for player $i$ is defined as:

$$
\mathcal{U}_{i}: \mid \begin{array}{ccc}
\mathcal{A}(t) \times \mathcal{H}_{t} & \longrightarrow & \mathbb{R} \\
\left(\underline{a}_{t}, \underline{h}_{t}\right) & \longmapsto & \mathcal{U}_{i}\left(\underline{a}_{t}, \underline{h}_{t}\right),
\end{array}
$$

where in the notation $X \longrightarrow Y, X$ refers to the set of arguments and $Y$ the set of images by the function and $x \longmapsto f(x)$ gives the mapping of each argument.

We may write $\mathcal{U}_{i}\left(\underline{a}_{t}, \underline{h}_{t}\right)$ as $\mathcal{U}_{i}\left(\underline{a}_{t, i}, \underline{a}_{t,-i}, \underline{h}_{t}\right)$. For clarity purposes, the notation $\mathbf{X}$ refers to a matrix whose $i$ th column is $\underline{X}_{i}$. The notation $\underline{x}$ refers to a vector whose $i$ th entry is $x_{i}$. We denote by $\{0,1\}^{x \times y}$ the set of matrices of dimension $x \times y$ containing only 0 s and $1 \mathrm{~s}$. We also use the notation $\{0,1\}^{x}$ to refer to the set $\{0,1\}^{x \times 1}$. The notation $\left[\underline{X}_{1}, \ldots, \underline{X}_{n}\right]$ refers to the matrix whose $i$ th column is the vector $\underline{X}_{i}$ and the notation $\mathbf{X}=\left[x_{i j}\right]$ refers to a matrix $\mathbf{X}$ whose $i$ th row and $j$ column is the element $x_{i j}$. The game $\mathcal{G}$ is said to be finite if the number of times it is played is finite. For such games, let $T$ be the final stage of the game.

\section{B. Potential Games}

Potential games have been introduced in [26]. The definition of a potential game $\mathcal{G}$ is given by: 
Definition 1. The game $\mathcal{G}$ is an exact potential game if there exists a function $\phi$ such that:

$$
\begin{gathered}
\forall i \in \mathcal{M}, \forall t, \forall \underline{a}_{t}, \underline{a}_{t}^{\prime} \in \mathcal{A}(t), \\
\mathcal{U}_{i}\left(\underline{a}_{t}, \underline{h}_{t}\right)-\mathcal{U}_{i}\left(\underline{a}_{t, i}^{\prime}, \underline{a}_{t,-i}, \underline{h}_{t}\right)= \\
\phi\left(\underline{a}_{t}, \underline{h}_{t}\right)-\phi\left(\underline{a}_{t, i}^{\prime}, \underline{a}_{t,-i}, \underline{h}_{t}\right) .
\end{gathered}
$$

In other words, a game $\mathcal{G}$ is an exact potential game if there is a function $\phi$ that measures exactly the difference in the utility due to unilaterally deviation of each player [24].

Such a function $\phi$ is called the exact potential of the game. Note that such potential does not directly guarantee the Pareto optimality of the Nash Equilibrium (see Cournot oligopolies [26]). Both Pareto optimality and Nash Equilibrium will be defined in the next section. Instead of being a warranty of Pareto efficiency, the potential function can be seen as a quantification of the disagreement among the players [26]. In the dynamic system [27], the potential represents a Lyapunov function of the game.

Note that other type of potential games can be found in the literature: the weighted, ordinal, generalized and best-response potential games [28]. The theorems stated in the next section will not depend on the nature of the potential game considered and hold for all of them [24] as far as the game is potential.

\section{Equilibrium Existence and Pareto Optimality}

The definition of the Pure Nash Equilibrium (NE) is the following:

Definition 2. An action profile $\underline{a}_{t}^{*} \in \mathcal{A}(t)$ is called a Pure Nash equilibrium if:

$$
\forall i \in \mathcal{M}, \mathcal{U}_{i}\left(\underline{a}_{t}^{*}, \underline{h}_{t}\right)=\max _{\underline{a}_{t, i} \in \mathcal{A}_{i}(t)} \mathcal{U}_{i}\left(\underline{a}_{t, i}, \underline{a}_{t,-i}^{*}, \underline{h}_{t}\right) .
$$

In other words, a NE is an action profile $\underline{a}_{t}^{*}$ in which no player can increase his utility by unilateral deviation. In engineering system, the NE is a stable point to operate [29].

An attractive property of the NE is called the ParetoOptimum Nash Equilibrium (PONE) which is defined as:

Definition 3. An action profile $\underline{a}_{t}^{*} \in \mathcal{A}(t)$ is called a ParetoOptimum Nash Equilibrium if for all NE action profile $\underline{b}_{t}^{*}$, we have:

$$
\forall i \in \mathcal{M}, \mathcal{U}_{i}\left(\underline{a}_{t}^{*}, \underline{h}_{t}\right) \geq \mathcal{U}_{i}\left(\underline{b}_{t}^{*}, \underline{h}_{t}\right) .
$$

In other words, the PONE is the NE that achieves the highest utility for all the players among all the other NE.

General results about equilibrium existence and uniqueness are provided in [30]. Since our problem is a cooperative control game, then we can make use of the results of the potential games [31]. Before stating the main results concerning equilibrium of potential games, we first define the coordination game [27]:

Definition 4. Let $\mathcal{G}=\left(\mathcal{M},\left\{\overline{\mathcal{A}}_{i}\right\}_{i}, \Omega,\left\{\mathcal{A}_{i}\right\}_{i},\left\{\alpha_{i}\right\}_{i}, q,\left\{\mathcal{U}_{i}\right\}_{i}\right)$ be a potential game with a potential function $\phi$. The game $\mathcal{G}^{\prime}=\left(\mathcal{M},\left\{\overline{\mathcal{A}}_{i}\right\}_{i}, \Omega,\left\{\mathcal{A}_{i}\right\}_{i},\left\{\alpha_{i}\right\}_{i}, q, \phi\right)$ is called the coordination game of $\mathcal{G}$.
The following theorem gives the relationship in terms of equilibrium between the potential game and its associated coordination game:

Theorem 1. Let $\mathcal{G}$ be a potential game with potential $\phi$ and $\mathcal{G}^{\prime}$ its associated coordination game. Then the set of NEs of $\mathcal{G}$ coincides with the set of NEs of $\mathcal{G}^{\prime}$. Moreover, the actions profile $\underline{a}_{t}^{*} \in \mathcal{A}(t)$ maxima of $\phi$ are $N E$ of $\mathcal{G}$.

Proof: The proof of this theorem can be found in [27].

Note that the converse of this theorem is not generally true i.e. not all the NEs of $\mathcal{G}$ are maxima of $\phi$ [27]. The existence of a NE in potential game is ensured by this corollary:

Corollary 1. Every finite potential game admits at least one $N E$.

Proof: The proof comes directly from Theorem 11. For a finite game, the potential function is finite and therefore have at least one maximum and thus the game admits at least one NE.

The following theorem characterize the PONE in a coordination game:

Theorem 2. Let $\mathcal{G}$ be a potential game with potential $\phi$ such that its corresponding coordination game is itself i.e. $\mathcal{G}=\mathcal{G}^{\prime}$ then the maximum of $\phi$ is the PONE of $\mathcal{G}$.

Proof: According to Theorem 1 the maximum $\underline{a}_{t}^{*}$ of $\phi$ is a NE of $\mathcal{G}$ and since $\phi$ is the utility function of $\mathcal{G}$ therefore $\underline{a}_{t}^{*}$ is a NE that yields the highest utility. More specifically $\underline{a}_{t}^{*}$ yields the highest utility among all the NE of $\mathcal{G}$ and thus it is the PONE.

\section{Network Model AND Protocol}

\section{A. Network Model}

The network we consider in this paper consists of a set $\mathcal{M}=\{1, \ldots, M\}$ geographically close clients (players) that require the reception of source packets that the base station (BS) holds. Each player is interested in receiving the frame $\mathcal{N}=\{1, \ldots, N\}$ of source packets regardless of the order.

In the first $n$ time slots, the BS broadcasts the $N$ source packets of the frame $\mathcal{N}$ uncoded. Each player $i$ is experiencing a packet erasure probability $q_{i}$ assumed to be constant during this phase. Each player listens to the transmitted packets and sends an acknowledgement (ACK) upon each successful reception of each packet. We assume that at the end of this initial phase, each packet of the frame is at least acknowledged by one of the players. Otherwise, this packet is re-transmitted by the BS.

After this initialization phase, for each player $i$, the packets of the frame $\mathcal{N}$ can be in one of the following sets:

- The Has set (denoted by $\mathcal{H}_{i}$ ): The sets of packets successfully received by player $i$.

- The Wants set (denoted by $\mathcal{W}_{i}$ ): The sets of packets that were erased at player $i$. Clearly, we have $\mathcal{W}_{i}=\mathcal{N} \backslash \mathcal{H}_{i}$.

In this configuration, we assume a perfect reception of the acknowledgement by all the players and that each player 
knows the packets sets of all the other players. Each player stores the information obtained after the transmission at time $(t-1)$ in a state matrix $(\mathrm{SM}) \mathbf{S}(t)=\left[s_{i j}(t)\right], \forall i \in \mathcal{M}, \forall j \in$ $\mathcal{N}$ such that:

$$
s_{i j}(t)= \begin{cases}0 & \text { if } j \in \mathcal{H}_{i}(t) \\ 1 & \text { if } j \in \mathcal{W}_{i}(t) .\end{cases}
$$

\section{B. Network Protocol}

After the initial transmission, the recovery phase starts. In this phase, the players cooperate to recover their missing packets by transmitting to each other binary XOR encoded packets of the source packets they already hold in order to minimize the completion time.

The packet combination is chosen according to the available packets they have, the information available in the SM and the expected erasure patterns of the links. Let $\mathbf{P}=\left[p_{i j}\right], i, j \in \mathcal{M}$ denote the packet erasure probability (i.e. the probability to loss a packet) from player $j$ to player $i$. All the packet erasure probabilities are assumed to be constant during the transmission of the frame. Since the packet erasure probability depends not only on the link but also on the available power used to transmit, therefore $p_{i j}$ can be different from $p_{j i}$. We assume that each player knows all the packet erasure probabilities linking him to other players (i.e. player $i$ knows $\left.p_{j i}, \forall j \in \mathcal{N}\right)$ and that each transmission can be heard by all the players. Therefore only one player will transmit a packet combination at each time slot. Otherwise, due to interference between transmissions, none of the players will be able to decode a packet.

In this phase, the transmitted coded packets can be one of the following three options for each player $i$ :

- Instantly Decodable: A packet is instantly decodable for player $i$ if the encoded packet contain at most one packet the player does not have so far. In other words, it contains only one packet from $\mathcal{W}_{i}$.

- Non-Instantly Decodable: A packet is non instantly decodable for player $i$ if it contains more than one packet missing for that player. In other words, it contains at least two packets from $\mathcal{W}_{i}$.

- Non-innovative: A packet is non-innovative for player $i$ if it do not allow him to reduce its Wants set. In other words, it does not contains packets from $\mathcal{W}_{i}$.

We define the conventional decoding delay [8], [9] as follows:

Definition 5. At any cooperative phase transmission, a player $i$, with non-empty Wants set, experiences a one unit increase of decoding delay if it successfully receivers a packet that is either non-innovative or non-instantly decodable.

The cooperation decoding delay can be defined as:

Definition 6. At any cooperative phase transmission, a player $i$, with non-empty Wants set, experiences a one unit increase of decoding delay if not exactly one player transmitted or only one player transmitted and its conventional decoding delay increases.
In other words, if more than one or none players transmits, all the players will experience a decoding delay and if player $i$ is the only transmitting player, he will experience a delay along with all players that successfully received a packet that is either non-innovative or non-instantly decodable. In the rest of the paper, we will use the term decoding delay to refer to the cooperative decoding delay. We define the targeted players by a transmission as the players that can instantly decode a packet from that transmission. After each transmission in the recovery phase, its targeted players send acknowledgements consisting of one bit indicating the successful reception. This process is repeated until all players report that they obtained all the packets. Let $T$ be final stage of the game. The definition of $T$ can be written as:

$$
T=\min \left\{t \in \mathbb{N}^{*} \text { such that } \mathbf{S}(t)=\mathbf{0}\right\} .
$$

Since we assume single hop transmissions, which means that all the players are in the transmission range of each other, each of them can already overhear all the feedback sent by the other players and thus the system does not require any additional feedback load.

\section{Completion Time Game Formulation}

In this section, we first introduce the game parameter to be able to model the problem of minimizing the completion time in IDNC as a non-cooperative potential game. We then provide the expression of the utility for the game.

\section{A. Game Parameters}

Let $\underline{\kappa}^{i}(t)$ be the optimal packet combination that player $i$ can generate at the stage $t$ of the game. We have $\underline{\kappa}^{i}(t) \in$ $\{0,1\}^{N}$ with $\kappa_{j}^{i}(t)=1$ means that packet $j$ is included in the packet combination and 0 otherwise. The mathematical expression of this combination can be found in [22]. Since the SM is known by all players, therefore each player can compute the optimal packet combination (or a sub-optimal since the computation of the optimal was shown to be NPhard) of all the other players.

In this game formulation, we assume complete and perfect information. The former assumption means that the actions available to the players and the utility functions are common knowledge i.e. every player knows the data of the game, every player knows that the other players know the data of the game, every player knows that every player knows that every players knows the data of the game, and so on, ad infinitum. The latter assumption means all the players know the history of the game perfectly. Note that these assumptions do not add extra constraints to the problem but are rather intrinsic to it.

At each stage of the game, each player has two possible actions: either he transmits or he listens. Therefore, we define the action space of player $i$ at each stage $t$ of the game as $\mathcal{A}_{i}(t)=\left\{\right.$ transmit $\underline{\kappa}^{i}(t)$, remain silent $\}$. Note that the action space of the players are not symmetric since each player can transmit a different packet combination at each stage. Let $\underline{a}_{t}$ be the actions taken by all the users at the stage $t$. For simplicity 
of notation, we will define $\underline{a}_{t}$ as the following:

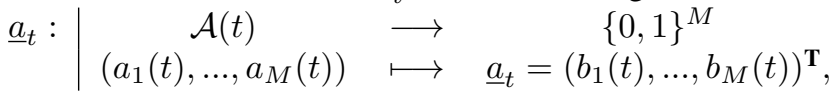

where,

$$
b_{i}(t)= \begin{cases}0 & \text { if } a_{i}(t)=\{\text { remain silent }\} \\ 1 & \text { otherwise }\end{cases}
$$

The set of targeted players by a packet combination are those that can instantly decode an innovative packet from the combination. Let $\underline{\tau}_{\underline{\kappa}(t)}$ be the set of targeted player by the packet combination $\underline{\kappa}$ at the stage $t$ of the game. The mathematical definition of this set is given by:

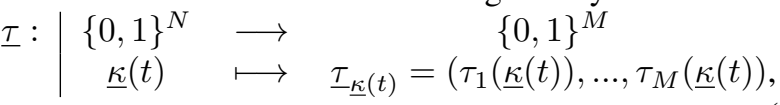

where:

$$
\tau_{i}(\underline{\kappa}(t))= \begin{cases}1 & \text { if } \sum_{j=1}^{N} s_{i j}(t) \kappa_{j}(t)=1 \\ 0 & \text { otherwise }\end{cases}
$$

The players that experience a conventional decoding delay after a transmission are those with non-empty Wants set and are not targeted by the transmission. Define $\overline{\bar{\tau}}_{\kappa(t)}=\underline{1}_{-}-\underline{\tau}_{\underline{\kappa}(t)}$ as the set of non targeted players and let $\underline{M}^{w}(t)$ be the set of players with non-empty Wants set defined as follows:

$$
\underline{M}_{i}^{w}(t)= \begin{cases}0 & \text { if } \sum_{j=1}^{N} s_{i j}(t)=0 \\ 1 & \text { otherwise. }\end{cases}
$$

The state $\boldsymbol{\omega}$ of the game is the erasure patterns of the links between each couple of players. These states can be described by the following formula:

$$
\begin{array}{c|ccc}
\boldsymbol{\omega}: & \mathbb{N}^{*} & \longrightarrow & \Omega=\{0,1\}^{M \times M} \\
t & \longmapsto & \boldsymbol{\omega}(t)=\left[X_{i j}\right], 1 \leq i, j \leq M,
\end{array}
$$

where $X_{i j}$ is a Bernoulli random variable defined as follows:

$$
X_{i j}= \begin{cases}0 & \text { with probability } p_{i j} \\ 1 & \text { with probability } 1-p_{i j} .\end{cases}
$$

The $X_{i j}$ are independent of each other and therefore the $\boldsymbol{\omega}(t)$ are independent identically distributed (iid). The game can be seen as a random matrix game. We now can compute the decoding delay $\underline{\mathcal{D}}_{w, \kappa}$ experienced by all the users when user $i$ sends the packet combination $\kappa$ at the stage $t$ using the following expression:

$$
\underline{\mathcal{D}}_{w_{i}, \kappa}(t): \mid \begin{array}{ccc}
\{0,1\}^{M+N} & \longrightarrow & \{0,1\}^{M} \\
\left(\underline{\omega}_{i}(t), \underline{\kappa}(t)\right) & \longmapsto & \underline{\omega}_{i}(t) \circ \underline{\bar{\tau}}_{\underline{\kappa}(t)} \circ \underline{M}^{w}(t),
\end{array}
$$

where $\circ$ is the Hadamard product. We also define the cumulative decoding delay experienced by all players since the beginning of the recovery phase (beginning of the game) until the stage $t$ of the game:

$$
\underline{\mathbb{D}}: \mid \begin{array}{ccc}
\{0,1\}^{M} \times \mathcal{H}_{t} & \longrightarrow & \{0,1\}^{M} \\
\left(\underline{a}_{t}, \underline{h}_{t}\right) & \longmapsto & \underline{\mathbb{D}}\left(\underline{a}_{t}, \underline{h}_{t}\right),
\end{array}
$$

where:

$$
\begin{aligned}
& \underline{\mathbb{D}}\left(\underline{a}_{t}, \underline{h}_{t}\right)= \\
& \begin{cases}\underline{\mathbb{D}}\left(\underline{a}_{t-1}, \underline{h}_{t-1}\right)+\underline{M}^{w}(t) & \text { if }\left\|\underline{a}_{t}\right\|_{1} \neq 1 \\
\underline{\mathbb{D}}\left(\underline{a}_{t-1}, \underline{h}_{t-1}\right)+\underline{\mathcal{D}}_{w_{i}, \kappa^{i}}(t) & \text { otherwise } \\
\text { with } i \text { such that } a_{i}(t)=\left\|\underline{a}_{t}\right\|_{1} . & \end{cases}
\end{aligned}
$$

In the case of the Point to Multipoint (PMP) recovery process (when only the base station is transmitting), the completion time $\mathcal{C}_{i}$ can be approximated [22] by the following expression:

$$
\tilde{\mathcal{C}}_{i}=\frac{\mathcal{W}_{i}+\mathbb{D}_{i}-q_{i}}{1-q_{i}}
$$

Since in the CDE, all users may be transmitting to each other, then the completion time can be approximated by:

$$
\mathcal{C}_{i}=\frac{\mathcal{W}_{i}+\mathbb{D}_{i}-\bar{p}_{i}}{1-\bar{p}_{i}}
$$

where $\bar{p}_{i}$ is the average erasure probability linking the player $i$ to the other players. This average erasure probability can be expressed in terms of the erasure matrix as follows: $\bar{p}_{i}=$ $\frac{\left\|\underline{P}_{i}\right\|_{1}}{M}$. Let $\underline{\mathcal{C}}$ be the vector of the completion times, $\underline{\mathcal{W}}$ the vector of the Wants sets and $\bar{p}$ the one of the average erasures. Therefore, we define the expected completion time of each player as follows:

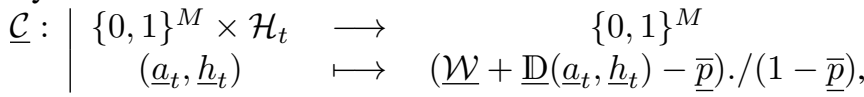

where the operator $\underline{x} . / y$ refers to the division of each element of vector $\underline{x}$ by the element of vector $\underline{y}$.

\section{B. Utility Functions}

In this first formulation, we take the cost (-utility) function to be the natural delay in the network.

\section{Game 1 (Completion time Game):}

- Players: Users in set $\mathcal{M}$

- History : $\underline{h}_{t}=$ Channel realization $\boldsymbol{\omega}(t)$ and players' action $\underline{a}_{t}$ at each stage $t \geq 1$.

- Strategies: Contingency plans for selection transmission policy at each stage $t \geq 1$ and for any given history $\underline{h}_{t}$.

- Utilities: $\mathcal{U}_{i}^{C T}$ for each player $i$, where at each stage $t \geq 1$ and for any given history $\underline{h}_{t}$ and action profile $\underline{a}_{t}$ :

$$
\mathcal{U}_{i}^{C T}: \mid \begin{array}{ccc}
0,1\}^{M} \times \mathcal{H}_{t} & \longrightarrow & \mathbb{R} \\
\left(\underline{a}_{t}, \underline{h}_{t}\right) & \longmapsto & \left.-\| \underline{\mathcal{C}}_{(} \underline{a}_{t}, \underline{h}_{t}\right) \|_{\infty} .
\end{array}
$$

This game formulation is a non-cooperative stochastic potential game. However, this definition of the game suffers from many flaws. First, by inspection of the NE of the game (Appendix A), we clearly can see that most of the NE yield the worst payoff. This occurs when more than one player is transmitting. Without a punishment policy, the game can loop infinitely without reducing the Wants set of any player. Secondly, this multitude of NE will not make the system work in its best point which is the PONE. For these reasons, the overall performance of the game will be very poor and a more robust definition of the game must be addressed. 


\section{Punishment and Pareto Optimality}

\section{A. Punishment and Back-off Function}

In the first definition of the game, after a collision occurs, each of the players that transmitted can re-transmit in the next stage of the game. In order to overcome the scenario in which multiple consecutive collisions occur, we impose a punishment period of $V$ to every player responsible of a collision. In other words, players responsible of a collision will back-off and will not be able to transmit during the next $V$ transmissions. Let $\underline{c}_{t}=\left(c_{1}(t), \ldots, c_{M}(t)\right)^{\mathbf{T}} \in\{0,1\}^{M}$ be the collision indicator defined as follows:

$$
c_{i}(t)= \begin{cases}1 & \text { if } a_{i}(t)=1 \text { and }\left\|\underline{a}_{t}\right\|_{1}>1 \\ 0 & \text { otherwise. }\end{cases}
$$

Let $\mathbf{C}$ be the collision history over the last $V$ stage of the game. The mathematical definition of this variable is:

$$
\begin{aligned}
& \text { C : } \mid \mathcal{H}_{t} \longrightarrow\{0,1\}^{M \times V} \\
& \underline{h}_{t} \longmapsto \mathbf{C}\left(\underline{h}_{t}\right)=\left[\underline{c}_{t-V}, \ldots, \underline{c}_{t-1}\right] .
\end{aligned}
$$

For notation consistency, the collision indicator for a non positive time index is taken 0 i.e. $\underline{c}_{-t}=\underline{0}, \forall t \geq 0$. The back-off function indicates at each stage $t$ of the game which players are allowed to transmit. The mathematical definition of this function is:

$$
\underline{\mathcal{B}}: \mid \begin{array}{ccc}
\{0,1\}^{M \times V} & \longrightarrow & \{0,1\}^{M} \\
\mathbf{C}\left(\underline{h}_{t}\right) & \longmapsto & \underline{\mathcal{B}}\left(\underline{h}_{t}\right)=\mathbf{C}\left(\underline{h}_{t}\right) \underline{1} .
\end{array}
$$

Let $\mathcal{A}_{i}^{\prime}(t)$ be the action space of player $i$ at each stage $t$ of the game defined as follows:

$$
\mathcal{A}_{i}^{\prime}(t)= \begin{cases}\mathcal{A}_{i}(t) & \text { if } \underline{\mathcal{B}}_{i}\left(\underline{h}_{t}\right)=0 \\ \{\text { remain silent }\} & \text { otherwise }\end{cases}
$$

\section{B. New Game Formulation}

In order to encourage sparsity of the action profile vector $\underline{a}_{t}$, the $\ell_{1}$ regularizer is added to the previous definition of the utility function. Moreover, prioritization between players is added when all action profiles will yield a higher expected completion time than in the previous stage of the game. This additional term is scaled by the number of player to not change the original game. The new game formulation is:

Game 2 (New completion time Game):

- Players: Users in set $\mathcal{M}$

- History : $\underline{h}_{t}=$ Channel realization $\boldsymbol{\omega}(t)$ and players' action $\underline{a}_{t}$ at each stage $t \geq 1$.

- Strategies: Contingency plans for selection of a transmission policy at each stage $t \geq 1$ and for any given history $\underline{h}_{t}$.

- Utilities: $\mathcal{U}_{i}^{C T}$ for each player $i$, where at each stage $t \geq 1$ and for any given history $\underline{h}_{t}$ and action profile $\underline{a}_{t}$ :

$$
\begin{aligned}
\mathcal{U}_{i}^{C T}\left(\underline{a}_{t}, \underline{h}_{t}\right) & =-\left\|\underline{\mathcal{C}}\left(\underline{a}_{t}, \underline{h}_{t}\right)\right\|_{\infty}-\left\|\underline{a}_{t}\right\|_{1} \\
& -\frac{\left\|\underline{\mathbb{D}}\left(\underline{a}_{t}, \underline{h}_{t}\right)-\underline{\mathbb{D}}\left(\underline{a}_{t-1}, \underline{h}_{t-1}\right)\right\|_{1}}{M} .
\end{aligned}
$$

As for Game 1, Game 2 is a non-cooperative stochastic potential games. In the next section of the game, we will proof that this formulation effectively addresses the concerns of the first version.

\section{GAME Equilibrium ANALYSis AND Distributed LEARNING ALGORITHM}

In virtue of Corollary 1, there exist at least one NE. Moreover, according to Theorem 2, the maximum of the utility function is the PONE of the game. However, the existence of the NE or the PONE is not sufficiency. We first define the price of anarchy, introduced in [32] to be able to characterize the equilibrium in a game.

Definition 7. The price-of-anarchy (PoA), at stage $t$, is the worst-case efficiency of a Nash Equilibrium among all possible strategies. In other words, the PoA is defined as:

$$
\operatorname{Po} A(t)=\frac{\max _{s \in S(t)} W(s)}{\min _{s \in E(t)} W(s)},
$$

where:

- $S(t)$ is the set of all possible strategies at stage $t$,

- $E(t)$ is the set of all NE at stage $t$,

- $W: S \longrightarrow \mathbb{R}$ is a fairness function .

The PoA is a concept that measures how the efficiency of a game degrades due to selfish behavior of its players. Since we have in our game the Pareto Equilibrium is a Nash equilibrium and the utility function is the same for all the players (the corresponding coordination game is the game itself), then the previous definition reduces to the following:

$$
\operatorname{PoA}(t)=\frac{\max _{s \in E(t)} \phi(s)}{\min _{s \in E(t)} \phi(s)} .
$$

The utility function is always negative in our context. Therefore, the PoA is a well defined quantity. In this paper, since the utility function is strictly negative, we will compute the PoA using the cost function $\phi^{\prime}=-\phi$. The PoA can be expressed in terms of the cost function as follows:

$$
\operatorname{PoA}(t)=\frac{\min _{s \in E(t)} \phi^{\prime}(s)}{\max _{s \in E(t)} \phi^{\prime}(s)} \text {. }
$$

\section{A. Game Equilibrium Analysis of Game 1}

The following theorem gives the PoA of the Game 1:

Theorem 3. The PoA of Game 1 can be expressed as follows:

$$
\begin{aligned}
& \operatorname{PoA}(t)= \\
& \begin{cases}1 & \text { if } Z(t)=\varnothing \\
1-\frac{Y_{0}(t)-\min _{j \in Z(t)}\left(Y_{j}(t)\right)}{\phi^{\prime}\left(\underline{a}_{t-1}, \underline{h}_{t-1}\right)+Y_{0}(t)} & \text { otherwise, }\end{cases}
\end{aligned}
$$

where $\phi^{\prime}=-\mathcal{U}_{i}^{C T}, \forall i \in \mathcal{M}$ is the cost of the game and the set $Z$ is the set defined by

$$
Z(t)=\left\{j \in \mathcal{M} \text { such that } Y_{j}(t)<Y_{0}(t)\right\},
$$

and $Y_{0}(t)=\max _{i \in Q(t)} \frac{1}{1-\bar{p}_{i}}$ is the increase in the cost function when not exactly one player is transmitting and $Y_{j}(t)=$ $\max _{i \in Q(t) \cap \underline{\mathcal{D}}_{w_{j}, \kappa^{j}}(t)} \frac{1}{1-\bar{p}_{i}}$ the cost when only player $j$ is trans- 
mitting with $Q(t)$ defined as:

$$
\begin{aligned}
& Q(t)=\{i \in \mathcal{M} \text { such that } \\
& \mathcal{C}_{i}\left(\underline{a}_{t-1}, \underline{h}_{t-1}\right)+1 /\left(1-\bar{p}_{i}\right)>\left\|\underline{\mathcal{C}}\left(\underline{a}_{t-1}, \underline{h}_{t-1}\right)\right\|_{\infty} \\
& \left.\quad \text { and } M_{i}^{w}=1\right\} .
\end{aligned}
$$

Proof: The proof of this Theorem can be found in Appendix A.

\section{B. Equilibrium Investigation of Game 2}

The following theorem gives the PoA of the Game 2:

Theorem 4. The PoA of Game 6 can be expressed as follows: $P o A^{\prime}(t)=$

$$
\left\{\begin{array}{l}
\min _{\left\|\underline{a}_{t}\right\|_{1}=a_{i}(t)=1} \phi^{\prime}\left(\underline{a}_{t-1}, \underline{h}_{t-1}\right)+\frac{\left\|\underline{\mathcal{D}}_{\omega_{i}, \kappa^{i}}\right\|_{1}}{M}+1+Y_{i}(t) \\
\max _{\left\|\underline{a}_{t}\right\|_{1}=a_{i}(t)=1} \phi^{\prime}\left(\underline{a}_{t-1}, \underline{h}_{t-1}\right)+\frac{\left\|\underline{\mathcal{D}}_{\omega_{i}, \kappa^{i}}\right\|_{1}}{M}+1+Y_{i}(t) \\
\min _{\left\|\underline{a}_{t}\right\|_{1}=a_{i}(t)=1} \phi^{\prime}\left(\underline{a}_{t-1}, \underline{h}_{t-1}\right)+\frac{\left\|\underline{\mathcal{D}}_{\omega_{i}, \kappa^{i}}\right\|_{1}}{M}+1+Y_{0}(t) \\
\max _{\left\|\underline{a}_{t}\right\|_{1}=a_{i}(t)=1} \phi^{\prime}\left(\underline{a}_{t-1}, \underline{h}_{t-1}\right)+\frac{\left\|\underline{\mathcal{D}}_{\omega_{i}, \kappa^{i}}\right\|_{1}}{M}+1+Y_{0}(t) \\
\text { otherwise. }
\end{array}\right.
$$

where $\phi^{\prime}=-\mathcal{U}_{i}^{C T}, \forall i \in \mathcal{M}$ is the cost of the game and the set $Z$ is the set defined by

$$
Z(t)=\left\{j \in \mathcal{M} \text { such that } Y_{j}(t)<Y_{0}(t)\right\},
$$

and $Y_{0}(t)=\max _{i \in Q(t)} \frac{1}{1-\bar{p}_{i}}$ is the increase in the cost function when not exactly one player is transmitting and $Y_{j}(t)=$ $\max _{i \in Q(t) \cap \underline{\mathcal{D}}_{w_{j}, \kappa^{j}}(t)} \frac{1}{1-\bar{p}_{i}}$ the cost when only player $j$ is transmitting with $Q(t)$ defined as:

$$
\begin{aligned}
& Q(t)=\{i \in \mathcal{M} \text { such that } \\
& \mathcal{C}_{i}\left(\underline{a}_{t-1}, \underline{h}_{t-1}\right)+1 /\left(1-\bar{p}_{i}\right)>\left\|\underline{\mathcal{C}}\left(\underline{a}_{t-1}, \underline{h}_{t-1}\right)\right\|_{\infty} \\
& \left.\quad \text { and } M_{i}^{w}=1\right\} .
\end{aligned}
$$

Moreover, the PoA can be bounded by the following expressions:

$$
1 \geq P o A^{\prime}(t) \geq\left\{\begin{array}{c}
1+Y_{0}(t)-\min _{j \in Z(t)}\left(Y_{j}(t)\right) \\
\phi^{\prime}\left(\underline{a}_{t-1}, \underline{h}_{t-1}\right)+2+Y_{0}(t) \\
1-\frac{1}{\phi^{\prime}\left(\underline{a}_{t-1}, \underline{h}_{t-1}\right)+2+Y_{0}(t)} \\
\text { otherwise . }
\end{array}\right.
$$

Proof: The proof of this Theorem can be found in Appendix B.

For stage $t$ of the game with $Z(t)=\varnothing$, the expected completion time will increase anyway and thus the PoA do not have a practical signification in this case. In stage $t$ where $Z(t) \neq \varnothing$, we have:

$$
\operatorname{Po} A^{\prime}(t)>\operatorname{PoA}(t)
$$

This new definition of the game offers a more efficient equilibrium. Therefore, for any learning algorithm and a long running period, Game 2 will performs better in terms of delay than Game 1.

\section{Distributed Learning Algorithm}

In this paper, we will employ the best-response algorithm to simulate the system. In the original formulation, by Cournot [33], players choose their actions sequentially. At each time slot, a player selects the action that is the best response to the action chosen by the other players in the previous time slot. Since the state of the game is not known to players, the utility function will be replaced by the expected utility function. This can be done by replacing the actual state $\underline{\omega}_{i}(t)$ by its expected value $\underline{P}_{i}(t)$ in $(16)$. The following theorem characterize the outcome of the best-response algorithm for our games:

Theorem 5. For the second version of the game, the bestresponse algorithm will make the system operate in the PONE of the game.

Proof: To proof this theorem, we fist introduce the following theorem:

Theorem 6. Let $\mathcal{G}$ be a best-reply potential game with $\mathcal{V}$ a best response potential. If the action $\underline{a}_{t}^{*}$ maximizes $\mathcal{V}$, then $\underline{a}_{t}^{*}$ is a $N E$.

Proof: The proof of this theorem can be found in [34].

From our previous analysis, the NE of the second game are located on the one dimensional line in which only one player is transmitting. Let $\underline{a}_{t}^{*}$ be the PONE of the game such that $\left\|\underline{a}_{t}^{*}\right\|_{1}=a_{i}(t)=1$. Assume that the outcome of the bestresponse algorithm is the action profile $\underline{a}_{t}^{\prime} \neq \underline{a}_{t}^{*}$. In virtue of Theorem 6 and our previous analysis, the action profile will have $\left\|\underline{a}_{t}^{\prime}\right\|_{1}=a_{j}(t)=1, j \neq i$. For simplicity, assume that players take action sequentially in order. Assume first that $j<i$ i.e. player $j$ will take action before player $i$. Therefore, player $j$ did not take its best-response game because he can insure better payoff by choosing not to transmit. Hence, we obtain $j \geq i$. Assume now $j>i$. Since player $i$ is taken its best action then it will transmit. According to theorem 6 player $j$ is unable to transmit otherwise the outcome will not be a NE of the game. Therefore we obtain $i=j$. In other words, the only outcome of the best-response algorithm is the PONE of the game.

\section{Simulation Results}

In this section, we first present the simulation results comparing the delay encountered by players when applying the PMP system [22] against the distributed non decentralized cooperative data exchange. We, then, compare the delay experienced by clients against the player-player packet erasure probability relatively to the base station-player packet erasure probability since the short range communications are more reliable than the base station-player communications [20], [23. 


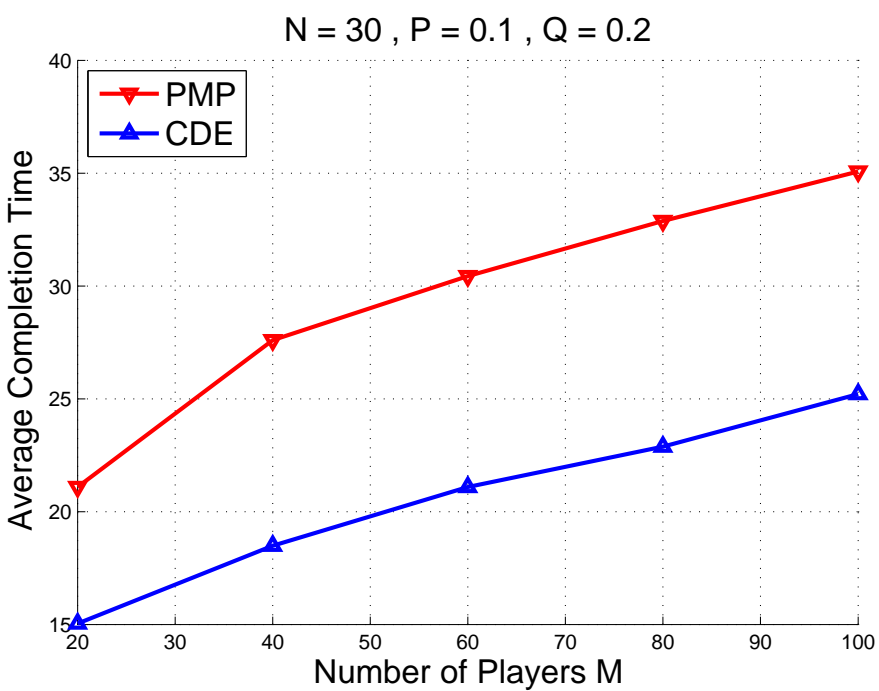

Fig. 1. Mean completion time versus number of players $M$.

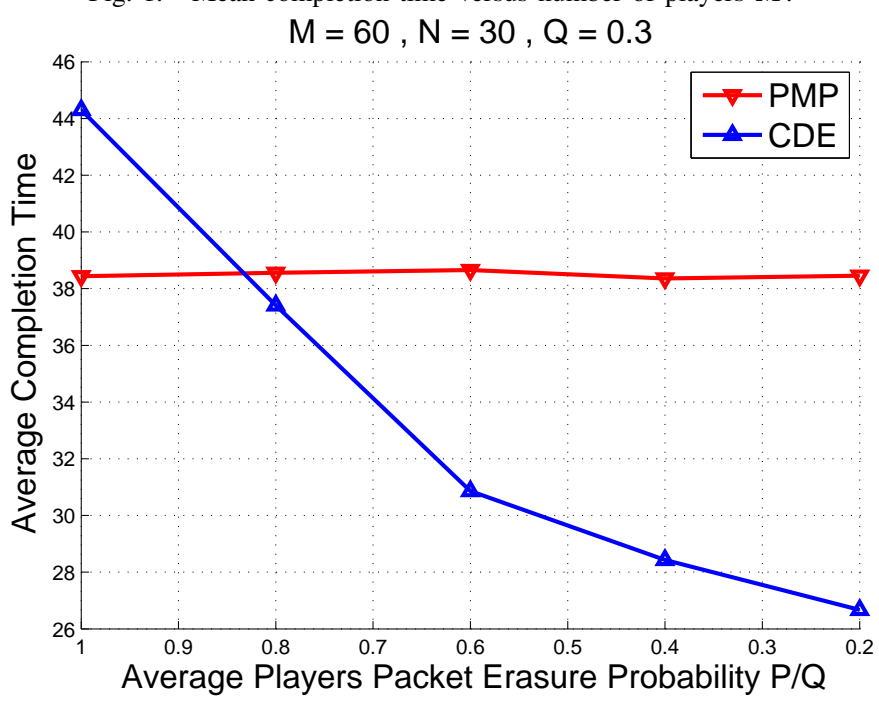

Fig. 2. Mean completion time versus players erasure $P$.

In these simulations, the delay is computed over a large number of iterations and the average value is presented. We assume that the packet erasure probability remains constant during a delivery period and change from iteration to iteration while keeping its mean, $P$ of the player-player and $Q$ for the base BS-players, constant. We also assume that each player have perfect knowledge of the packet erasure probabilities linking him to the other player (i.e. the estimation of this probability is perfect).

Figure 1 depicts the comparison of the average completion time achieved by the PMP scheme and our distributed CDE scheme against the number of players $M$ for $N=30, Q=$ 0.2 , and $P=0.1$. Figure 2 presents the mean completion time against the ratio of the player-player and BS-player erasure probability $P / Q$ for $M=60, N=30$, and $Q=0.3$.

From all the figures, we can clearly see that our cooperative data exchange algorithms outperform the traditional PMP approach. Figure 1, illustrates the gain in using a distributed algorithm when the player-player channel conditions is better than the BS-player channel $(P=0.5 Q)$.

Figure 2 illustrates the mean completion time against the player-player erasure probability for a fixed BS-player erasure. In this configuration, for the same erasure probability, the PMP scheme outperform the distributed approach. This can be explained first by the fact that the BS has all the packets whereas any one player has only a subset and thus, in general, the BS has better ability to form coding combinations that target more players than any single player. It can also be explained by the fact that in our CDE approach, the approximation of the completion time using the decoding delay approach requires the erasure probability between the sender in large sense and the player. For the PMP scheme only the base station is transmitting and therefore this probability is fixed (from BS to players). However in the CDE scheme, all players can transmit and we approximate the probability by the average erasure linking each player to all the other players. This approximation degrades the scheme. However as the channel linking players become better, CDE starts to outperform even if players send less efficient coding combinations and we clearly can see the difference between our scheme and the PMP one.

\section{Conclusion And Future Perspectives}

In this paper, we formulated the problem of minimizing the completion time of instantly decodable network coding for cooperative data exchange in decentralized wireless network as cooperative control game. We employed game theory as a tool to improve the distributed solution by overcoming the need for a central controller or additional signaling in the system. We modeled the session by self-interested players in a noncooperative potential game. The utility functions is designed in such way that increasing individual payoff results in a collective behavior achieving both a desirable system performance and the Pareto optimal solution. We compared our approach to the one of the conventional point-to-multipoint recovery process. Numerical results showed that our formulation largely outperforms the conventional PMP scheme in most practical scenarios and achieved a lower delay. The advantage of this formulation is that it can be easily extended. For example, this formulation can be extended to the case where not all the players are in the range of each other. In other words, the utility function will not be completely defined for the players. Another interesting research direction is the multicast cast with limited range. In this scenario, the packet demand of each player can differ and players are not all in the transmission range of each other. Finally, the case of imperfect feedback is another important and more practical extension.

\section{APPENDIX A PROOF OF THEOREM 3}

Note that the cost function can be written as:

$$
\phi^{\prime}\left(\underline{a}_{t}, \underline{h}_{t}\right)=\phi^{\prime}\left(\underline{a}_{t-1}, \underline{h}_{t-1}\right)+\xi\left(\underline{a}_{t}, \underline{h}_{t}\right),
$$

with:

$$
\xi\left(\underline{a}_{t}, \underline{h}_{t}\right)=\left\|\underline{\mathcal{C}}\left(\underline{a}_{t}, \underline{h}_{t}\right)\right\|_{\infty}-\left\|\underline{\mathcal{C}}\left(\underline{a}_{t-1}, \underline{h}_{t-1}\right)\right\|_{\infty} .
$$


Let $Q(t)$ be the set of players that can potentially increase the cost function at stage $t$ of the game. The mathematical definition of this set is the following:

$$
\begin{aligned}
& Q(t)=\{i \in \mathcal{M} \text { such that } \\
& \mathcal{C}_{i}\left(\underline{a}_{t-1}, \underline{h}_{t-1}\right)+1 /\left(1-\bar{p}_{i}\right)>\left\|\underline{\mathcal{C}}\left(\underline{a}_{t-1}, \underline{h}_{t-1}\right)\right\|_{\infty} \\
& \left.\quad \text { and } M_{i}^{w}=1\right\} .
\end{aligned}
$$

Clearly, if $Q(t)=\varnothing$, then any action profile $\underline{a}_{t}^{*} \in \mathcal{A}(t)$ is a NE since all the profiles will not change the cost function $\phi^{\prime}\left(\underline{a}_{t}^{*}, \underline{h}_{t}\right)=\phi^{\prime}\left(\underline{a}_{t-1}, \underline{h}_{t-1}\right)$. In that case, we have $\operatorname{PoA}(t)=$ 1 . Now assume $Q(t) \neq \varnothing$. For action profiles $\underline{a}_{t}$ the cost function will increase according to the norm of the action profile by the quantity:

$$
\begin{aligned}
& \phi^{\prime}\left(\underline{a}_{t}, \underline{h}_{t}\right)-\phi^{\prime}\left(\underline{a}_{t-1}, \underline{h}_{t-1}\right)= \\
& \begin{cases}\max _{i \in Q(t)} \frac{1}{1-\bar{p}_{i}} & \text { if }\left\|\underline{a}_{t}\right\|_{1} \neq 1 \\
\max _{i \in Q(t) \cap \underline{D}_{w_{j}, \kappa^{j}}(t)} \frac{1}{1-\bar{p}_{i}} & \text { if }\left\|\underline{a}_{t}\right\|_{1}=a_{j}(t)=1 .\end{cases}
\end{aligned}
$$

Let $Z(t)$ be the set of players that can target players in the critical set $Q(t)$ and reduce the increase in the cost function. This set is defined as:

$$
\begin{aligned}
& Z(t)=\{j \in \mathcal{M} \text { such that } \\
& \left.\qquad \max _{i \in Q(t) \cap \underline{D}_{w_{j}, \kappa^{j}}(t)} \frac{1}{1-\bar{p}_{i}}<\max _{i \in Q(t)} \frac{1}{1-\bar{p}_{i}}\right\} .
\end{aligned}
$$

Two cases can be distinguished:

- $Z(t)=\varnothing$ : all action profile $\underline{a}_{t}^{*}$ will yield the same cast function $\phi^{\prime}\left(\underline{a}_{t}^{*}, \underline{h}_{t}\right)=\phi^{\prime}\left(\underline{a}_{t-1}, \underline{h}_{t-1}\right)+\max _{i \in Q(t)} \frac{1}{1-\bar{p}_{i}}$. Therefore all action profile are NE of the game and the PoA is equal to 1 .

- $Z(t) \neq 0$ : action profile $\underline{a}_{t}^{*}=a_{j}(t)$ such that $j \in Z(t)$ will yield a lower cost function than the other profiles.

Define $Y_{0}(t)=\max _{i \in Q(t)} \frac{1}{1-\bar{p}_{i}}$ as the increase in the cost function when not exactly one player is transmitting and $Y_{j}(t)=\max _{i \in Q(t) \cap \underline{\mathcal{D}}_{w_{j}, \kappa^{j}}(t)} \frac{1}{1-\bar{p}_{i}}$ the increase when only player $j$ is transmitting. Clearly for action profiles $\underline{a}_{t}^{*}$ such that $\left\|\underline{a}_{t}^{*}\right\|_{1}=0$ are not NE since the unilateral deviation of player $i \in Z(t)$ will decrease the cost function. For action profile $\underline{a}_{t}^{*}$ such that $\left\|\underline{a}_{t}^{*}\right\|_{1}>2$, for any unilateral deviation, we have $\left\|\underline{a}_{t, i}, \underline{a}_{t,-i}^{*}\right\|>1$. Therefore, the cost function is unchanged and all these action profiles are NE. Let $\underline{a}_{t}^{*}$ be an action profile such that $\left\|\underline{a}_{t}^{*}\right\|_{1}=a_{j}(t)=1$, then the difference in the cost function for any unilateral deviation of player is:

$$
\begin{aligned}
& \phi^{\prime}\left(\underline{a}_{t, i}, \underline{a}_{t,-i}^{\prime} \underline{h}_{t}\right)-\phi^{\prime}\left(\underline{a}_{t}^{*}, \underline{h}_{t}\right) \\
& =\xi\left(\underline{a}_{t, i}, \underline{a}_{t,-i}^{*}, \underline{h}_{t}\right)-\xi\left(\underline{a}_{t}^{*}, \underline{h}_{t}\right)=Y_{0}(t)-Y_{j}(t) .
\end{aligned}
$$

Thus, any unilateral deviation will yield the same or a higher cost function. These profiles are NE of the game. Let the action profile be $\underline{a}_{t}^{*}$ with $\left\|\underline{a}_{t}^{*}\right\|_{1}=a_{i}(t)+a_{j}(t)=2$. Two scenarios can occur:

- $i \notin Z(t)$ and $j \notin Z(t)$ : By the same argument than for the case where $\left\|\underline{a}_{t}^{*}\right\|_{1}>2$, any unilateral deviation will yield the same cost $\phi^{\prime}\left(\underline{a}_{t}^{*}, \underline{h}_{t}\right)=\phi^{\prime}\left(\underline{a}_{t-1}, \underline{h}_{t-1}\right)+Y_{0}(t)$. Therefore it is a NE of the game.

- $i \in Z(t)$ or $j \in Z(t)$ : By the same argument than for the case $\underline{a}_{t}^{*}=\underline{0}$, the cost function can be decreased by unilateral deviation of player $i$ if $j \in Z(t)$ and inversely. This scenario is not a NE of the game.

We now characterize the set of all NE of the game:

$$
E(t)=\left\{\begin{array}{l}
\mathcal{A}(t) \text { if } Z(t)=\varnothing \\
E_{1}(t) \text { otherwise }
\end{array}\right.
$$

where

$$
\begin{aligned}
E_{1}(t) & =\left\{\underline{a}_{t} \in \mathcal{A}(t) \text { such that }\left\|\underline{a}_{t}\right\|_{1}=1 \text { or }\left\|\underline{a}_{t}\right\|_{1}>2\right. \\
& \text { or } \left.\left(\left\|\underline{a}_{t}\right\|_{1}=a_{i}(t)+a_{j}(t)=2 \text { and } i, j \notin Z(t)\right)\right\} .
\end{aligned}
$$

The $P o A$ of Game 3 can be expressed as follows:

$$
\begin{aligned}
& \operatorname{Po} A(t)= \\
& \begin{cases}1 & \text { if } Z(t)=\varnothing \\
1-\frac{Y_{0}(t)-\min _{j \in Z(t)}\left(Y_{j}(t)\right)}{\phi^{\prime}\left(\underline{a}_{t-1}, \underline{h}_{t-1}\right)+Y_{0}(t)} & \text { otherwise . }\end{cases}
\end{aligned}
$$

As for game 3, the utility function of Game 4 can be written as:

$$
\phi^{\prime}\left(\underline{a}_{t}, \underline{h}_{t}\right)=\phi^{\prime}\left(\underline{a}_{t-1}, \underline{h}_{t-1}\right)+\xi\left(\underline{a}_{t}, \underline{h}_{t}\right),
$$

with:

$$
\begin{aligned}
\xi\left(\underline{a}_{t}, \underline{h}_{t}\right)= & \left\|\underline{a}_{t}\right\|_{1}+\frac{\left\|\underline{\mathbb{D}}\left(\underline{a}_{t}, \underline{h}_{t}\right)-\underline{\mathbb{D}}\left(\underline{a}_{t-1}, \underline{h}_{t-1}\right)\right\|_{1}}{M} \\
& \left.+\| \underline{\mathcal{C}}_{a}, \underline{a}_{t}\right)\left\|_{\infty}-\right\| \underline{\mathcal{C}}\left(\underline{a}_{t-1}, \underline{h}_{t-1}\right) \|_{\infty} .
\end{aligned}
$$

Let $Q(t)$ be the set of players that can potentially increase the expected completion time at stage $t$ of the game. The mathematical definition of this set is the following:

$$
\begin{aligned}
& Q(t)=\{i \in \mathcal{M} \text { such that } \\
& \mathcal{C}_{i}\left(\underline{a}_{t-1}, \underline{h}_{t-1}\right)+1 /\left(1-\bar{p}_{i}\right)>\left\|\underline{\mathcal{C}}\left(\underline{a}_{t-1}, \underline{h}_{t-1}\right)\right\|_{\infty} \\
& \text { and } \left.M_{i}^{w}=1\right\} .
\end{aligned}
$$

Assume $Q(t)=\varnothing$, then any action profile $\underline{a}_{t}^{*} \in \mathcal{A}(t)$ will not increase the maximum decoding delay and we have:

$$
\begin{aligned}
\xi\left(\underline{a}_{t}, \underline{h}_{t}\right)=\left\|\underline{a}_{t}\right\|_{1}+\frac{\left\|\underline{\mathbb{D}}\left(\underline{a}_{t}, \underline{h}_{t}\right)-\underline{\mathbb{D}}\left(\underline{a}_{t-1}, \underline{h}_{t-1}\right)\right\|_{1}}{M} \\
= \begin{cases}\frac{\left\|\underline{M}^{w}(t)\right\|_{1}}{M} & \text { if }\left\|\underline{a}_{t}\right\|_{1}=0 \\
1+\frac{\left\|\underline{D}_{w_{i}, \kappa^{i}}(t)\right\|_{1}}{M} & \text { if }\left\|\underline{a}_{t}\right\|_{1}=a_{i}(t)=1 \\
\left\|\underline{a}_{t}\right\|_{1}+\frac{\left\|\underline{M}^{w}(t)\right\|_{1}}{M} & \text { otherwise. }\end{cases}
\end{aligned}
$$

Clearly, we can see that all the profiles $\underline{a}_{t}^{*}$ with $\left\|\underline{a}_{t}^{*}\right\|_{1}>1$ or $\left\|\underline{a}_{t}^{*}\right\|_{1}=0$ are not NE of the game anymore. Only action profile with one entry not equal to 0 are NE. Now assume $Q(t) \neq \varnothing$ and let $Z(t)$ be the set of players that can target players in the critical set $Q(t)$ and reduce the increase in the 
cost function. This set is defined as:

$$
\begin{aligned}
& Z(t)=\{j \in \mathcal{M} \text { such that } \\
& \left.\max _{i \in Q(t) \cap \underline{D}_{w_{j}, \kappa^{j}}(t)} \frac{1}{1-\bar{p}_{i}}<\max _{i \in Q(t)} \frac{1}{1-\bar{p}_{i}}\right\} .
\end{aligned}
$$

Two cases can be distinguished:

- $Z(t)=\varnothing:$ the expected completion time will increase by the same amount $\max _{i \in Q(t)} \frac{1}{1-\bar{p}_{i}}$ for all action profiles.

- $Z(t) \neq 0$ : some action profiles lead to lower increase of the expected completion time than others.

Define $Y_{0}(t)=\max _{i \in Q(t)} \frac{1}{1-\bar{p}_{i}}$ as the increase in the cost function when not exactly one player is transmitting and $Y_{j}(t)=\max _{i \in Q(t) \cap \underline{D}_{w_{j}, \kappa j}(t)} \frac{1}{1-\bar{p}_{i}}$ the increase when only player $j$ is transmitting. If $Z(t)=\varnothing$ for an action profile $\underline{a}_{t}^{*}$, the cost function can be expressed as:

$$
\begin{aligned}
& \xi\left(\underline{a}_{t}^{*}, \underline{h}_{t}\right)=Y_{0}(t) \\
& + \begin{cases}\frac{\left\|\underline{M}^{w}(t)\right\|_{1}}{M} & \text { if }\left\|\underline{a}_{t}\right\|_{1}=0 \\
1+\frac{\left\|\underline{D}_{w_{i}, \kappa^{i}}(t)\right\|_{1}}{M} & \text { if }\left\|\underline{a}_{t}\right\|_{1}=a_{i}(t)=1 \\
\left\|\underline{a}_{t}\right\|_{1}+\frac{\left\|\underline{M}^{w}(t)\right\|_{1}}{M} & \text { otherwise. }\end{cases}
\end{aligned}
$$

Clearly action profiles $\underline{a}_{t}^{*}$ of type $\left\|\underline{a}_{t}^{*}\right\|_{1} \neq 1$ are no more $\mathrm{NE}$ of the game. Now assume $Z(t) \neq \varnothing$ the utility varies with the chosen action profile. Let $\underline{a}_{t}^{*}$ be an action profile such that $\left\|\underline{a}_{t}^{*}\right\|_{1}=\alpha>1$. For some unilateral deviation of player that are transmitting, the cost function will decrease. In other words, we have:

$$
\begin{aligned}
& \phi^{\prime}\left(\underline{a}_{t, i}, \underline{a}_{t,-i}^{*}, \underline{h}_{t}\right)-\phi^{\prime}\left(\underline{a}_{t}^{*}, \underline{h}_{t}\right)= \\
& \left\{\begin{array}{c}
1>0 \quad \text { if }\left\|\underline{a}_{t, i}, \underline{a}_{t,-i}^{*}\right\|_{1}>1 \\
Y_{0}(t)-Y_{i}(t)+1+\frac{\left\|\underline{M}^{w}(t)\right\|-\left\|\underline{\mathcal{D}}_{w_{i}, \kappa^{i}}(t)\right\|_{1}}{M}>0 \\
\quad \text { if }\left\|\underline{a}_{t, i}, \underline{a}_{t,-i}^{*}\right\|_{1}=1 \text { and } i \in Z(t) \\
1+\frac{\left\|\underline{M}^{w}(t)\right\|-\left\|\underline{\mathcal{D}}_{w_{i}, \kappa^{i}}(t)\right\|_{1}}{M}>0 \\
\text { if }\left\|\underline{a}_{t, i}, \underline{a}_{t,-i}^{*}\right\|_{1}=1 \text { and } i \notin Z(t) .
\end{array}\right.
\end{aligned}
$$

Thus, all action profile $\underline{a}_{t}^{*}$ with $\left\|\underline{a}_{t}^{*}\right\|_{1}>1$ are not NE and it is clear to see that action profile $\underline{a}_{t}^{*}$ such that $\left\|\underline{a}_{t}^{*}\right\|_{1}=0$ are also not NE of the game. Let $\underline{a}_{t}^{*}$ be an action profile such that $\left\|\underline{a}_{t}^{*}\right\|_{1}=a_{i}(t)=1$ and $i \notin Z(t)$. The difference in the cost when player $i$ deviates is:

$$
\begin{aligned}
\phi^{\prime}\left(\underline{a}_{t}^{*}, \underline{h}_{t}\right)-\phi^{\prime}\left(\underline{a}_{t, i}, \underline{a}_{t,-i}^{*}, \underline{h}_{t}\right) \\
\quad=1+\frac{\left\|\underline{\mathcal{D}}_{w_{i}, \kappa^{i}}(t)\right\|_{1}-\left\|\underline{M}^{w}(t)\right\|}{M}>0 .
\end{aligned}
$$

Therefore such action profile is not a NE. Now consider the action profile $\underline{a}_{t}^{*}$ such that $\left\|\underline{a}_{t}^{*}\right\|_{1}=a_{i}(t)=1$ and $i \in Z(t)$.
The difference in the utility if any player deviates:

$$
\begin{gathered}
\phi^{\prime}\left(\underline{a}_{t, i}, \underline{a}_{t,-i}^{*}, \underline{h}_{t}\right)-\phi^{\prime}\left(\underline{a}_{t}^{*}, \underline{h}_{t}\right)= \\
\left\{\begin{array}{c}
1+Y_{0}(t)-Y_{i}(t)+\frac{\left\|\underline{M}^{w}(t)\right\|-\left\|\underline{\mathcal{D}}_{w_{i}, \kappa^{i}}(t)\right\|_{1}}{M}>0 \\
\text { if }\left\|\underline{a}_{t, j}, \underline{a}_{t,-j}^{*}\right\|_{1}=2 \\
Y_{0}(t)-Y_{i}(t)+\frac{\left\|\underline{M}^{w}(t)\right\|-\left\|\underline{\mathcal{D}}_{w_{i}, \kappa^{i}}(t)\right\|_{1}}{M}>0 \\
\text { if }\left\|\underline{a}_{t, j}, \underline{a}_{t,-j}^{*}\right\|_{1}=0 .
\end{array}\right.
\end{gathered}
$$

The set of all NE of the Game 4 can be expressed as:

$E(t)=$

$$
\begin{cases}\left\{\underline{a}_{t} \in \mathcal{A}(t) \text { s.t. }\left\|\underline{a}_{t}\right\|_{1}=a_{i}(t)=1, i \in Z(t)\right\} & \text { if } Z(t) \neq \varnothing \\ \left\{\underline{a}_{t} \in \mathcal{A}(t) \text { s.t. }\left\|\underline{a}_{t}\right\|_{1}=1\right\} & \text { otherwise. }\end{cases}
$$

The PoA of Game 4 can be expressed as follows:

$$
P o A^{\prime}(t)=
$$

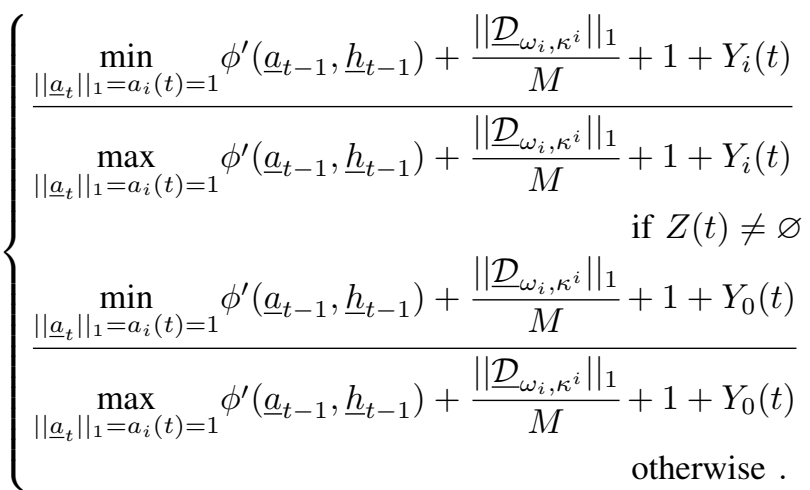

Moreover, the PoA can be bounded by the following expressions:

$$
1 \geq P o A^{\prime}(t) \geq\left\{\begin{array}{l}
1+Y_{0}(t)-\min _{j \in Z(t)}\left(Y_{j}(t)\right) \\
1-\frac{1}{\phi^{\prime}\left(\underline{a}_{t-1}, \underline{h}_{t-1}\right)+2+Y_{0}(t)} \\
1-\frac{\text { if } Z(t) \neq \varnothing}{\phi^{\prime}\left(\underline{a}_{t-1}, \underline{h}_{t-1}\right)+2+Y_{0}(t)} \\
\text { otherwise . }
\end{array}\right.
$$

\section{REFERENCES}

[1] R. Ahlswede, N. Cai, S.-Y. Li, and R. Yeung, "Network information flow," IEEE Transactions on Information Theory, vol. 46, no. 4, pp. 1204-1216, 2000

[2] X. Li, C.-C. Wang, and X. Lin, "On the capacity of immediatelydecodable coding schemes for wireless stored-video broadcast with hard deadline constraints," IEEE Transactions on Selected Areas in Communications, , vol. 29, no. 5, pp. 1094-1105, 2011.

[3] J.-S. Park, M. Gerla, D. Lun, Y. Yi, and M. Medard, "Codecast: A network-coding-based ad hoc multicast protocol," IEEE Tranaction on Wireless Communications, vol. 13, no. 5, pp. 76-81, 2006.

[4] T. Ho, R. Koetter, M. Medard, D. Karger, and M. Effros, "The benefits of coding over routing in a randomized setting," in Proc. of IEEE International Symposium on Information Theory, (ISIT' 2003), Yokohama, Japan, 2003, pp. 442-448.

[5] S. Katti, H. Rahul, W. Hu, D. Katabi, M. Medard, and J. Crowcroft, "Xors in the air: Practical wireless network coding," Proc. of ACM Computer Communication Review, (SIGCOMM' 2006), Pisa, Italy, October 2006, vol. 36, pp. 243-254. 
[6] S. Katti, D. Katabi, W. Hu, H. Rahul, and M. Medard, "The importance of being opportunistic: Practical network coding for wireless environments," in Proc. of 43rd Annual Allerton Conference on Communication, Control and Computing, (Allerton' 2005), Monticello, Illinois, USA, Sep. 2005.

[7] S. Rayanchu, S. Sen, J. Wu, S. Banerjee, and S. Sengupta, "Loss-aware network coding for unicast wireless sessions: design, implementation, and performance evaluation," in Proc. of the International Conference on Measurement and Modeling Of Computer Systems (ACM SIGMETRICS' 2008), Annapolis, MD, USA, ser. SIGMETRICS '08. New York, NY, USA: ACM, 2008, pp. 85-96.

[8] S. Sorour and S. Valaee, "Minimum broadcast decoding delay for generalized instantly decodable network coding," in Proc. of IEEE Global Telecommunications Conference, (GLOBECOM' 2010), Miami, Florida, USA, Dec. 2010, pp. 1-5.

[9] S. Sorour, N. Aboutorab, P. Sadeghi, M. S. Karim, T. Al-Naffouri, and M.-S. Alouini, "Delay reduction in persistent erasure channels for generalized instantly decodable network coding," Proc. of IEEE Vehicular Technology Conference, (VTC'2013), Dresden, Germany, pp. 1-5, June. 2013.

[10] P. Sadeghi, R. Shams, and D. Traskov, "An optimal adaptive network coding scheme for minimizing decoding delay in broadcast erasure channels," EURASIP Journal on Wireless Communications and Networking, vol. 2010, no. 1, 2010.

[11] A. Douik, S. Sorour, M.-S. Alouini, and T. Y. Al-Naffouri, "Delay reduction in lossy intermittent feedback for generalized instantly decodable network coding," in Proc. of IEEE 9th International Conference on Wireless and Mobile Computing, Networking and Communications, (WiMob' 2013), Lyon, France, Oct. 2013.

[12] L. Lu, M. Xiao, and L. Rasmussen, "Design and analysis of relay-aided broadcast using binary network codes," Journal of Communications (JCM), Special Issue on Advances in Communications and Networking, Nov. 2011.

[13] S. Sorour, A. Douik, S. Valaee, T. Y. Al-Naffouri, and M.-S. Alouini, "Partially blind instantly decodable network codes for lossy feedback environment," Accepted in IEEE Transactions on Wireless Communications, available ArXiv e-prints.

[14] A. Douik, S. Sorour, M.-S. Alouini, and T. Y. Al-Naffouri, "Delay minimization for instant decodable network coding in persistent channels with feedback intermittence," ArXiv e-prints, Jul. 2013.

[15] L. Lu, M. Xiao, and L. Rasmussen, "Relay-aided broadcasting with instantaneously decodable binary network codes," in Proc. of IEEE International Conference on Computer Communication Networks, (ICCCN), Maui, Hawaii, August, 2011.

[16] M. Esmaeilzadeh and P. Sadeghi, "Optimizing completion delay in network coded systems over tdd erasure channels with memory," in Proc. of IEEE International Symposium on Communications and Information Technologies, (ISCIT' 2012), Queensland, Australia, Oct. 2012, pp. 883888.

[17] A. Douik, S. Sorour, M.-S. Alouini, and T. Y. Al-Naffouri, Accepted in IEEE Wireless Communications Letters.

[18] S. Sorour and S. Valaee, "Effect of feedback loss on instantly decodable network coding," in Proc. of IEEE 7th International Wireless Communications and Mobile Computing Conference, (IWCMC' 2011), Istanbul, Turkey, July 2011, pp. 21-28.

[19] —, "On densifying coding opportunities in instantly decodable network coding graphs," in Proc. of IEEE International Symposium on Information Theory Proceedings, (ISIT' 2012), Cambridge, MA, USA, July 2012, pp. 2456-2460.

[20] N. Aboutorab, P. Sadeghi, and S. Tajbakhsh, "Instantly decodable network coding for delay reduction in cooperative data exchange systems," in Proc. of IEEE International Symposium on Information Theory Proceedings, (ISIT' 2013), Istanbul, Turkey, 2013, pp. 3095-3099.

[21] S. Sorour and S. Valaee, "On minimizing broadcast completion delay for instantly decodable network coding," in Proc. of IEEE International Conference on Communications, (ICC'2010), Cape Town, South Africa, May, 2010, pp. 1-5.

[22] A. Douik, S. Sorour, M.-S. Alouini, and T. Y. Al-Naffouri, "Completion Time Reduction in Instantly Decodable Network Coding Through Decoding Delay Control," ArXiv e-prints, Jan. 2014.

[23] S. Tajbakhsh and P. Sadeghi, "Coded cooperative data exchange for multiple unicasts," in Proc. of IEEE Information Theory Workshop, (ITW' 2012), Lausanne, Switzerland, 2012, pp. 587-591.
[24] S. Lasaulce and H. Tembine, Game Theory and Learning for Wireless Networks: Fundamentals and Applications, 1st ed. Academic Press, 2011.

[25] J. Filar and K. Vrieze, Competitive Markov Decision Processes. Springer, 1997.

[26] D. Monderer and L. S. Shapley, "Potential games," Games and Economic Behavior, vol. 14, no. 1, pp. 124 - 143, 1996. [Online]. Available: http://www.sciencedirect.com/science/article/pii/S0899825696900445

[27] G. Scutari, S. Barbarossa, and D. Palomar, "Potential games: A framework for vector power control problems with coupled constraints," in Proc. of IEEE International Conference on Acoustics, Speech and Signal Processing, (ICASSP' 2006), Toulouse, France., vol. 4, May 2006.

[28] M. Voorneveld, "Best-response potential games," Economics Letters, vol. 66, no. 3, pp. 289 - 295, 2000. [Online]. Available: http://www.sciencedirect.com/science/article/pii/S0165176599001962

[29] G. Arslan, J. R. Marden, and J. S. Shamma, "Autonomous vehicletarget assignment: A game-theoretical formulation," Journal of Dynamic Systems, Measurement, and Control, vol. 129, no. 5, pp. pp. 584-596, 2007.

[30] J. B. Rosen, "Existence and uniqueness of equilibrium points for concave n-person games," Econometrica, vol. 33, no. 3, pp. pp. 520-534, 1965. [Online]. Available: http://www.jstor.org/stable/1911749

[31] J. Marden, G. Arslan, and J. Shamma, "Cooperative control and potential games," IEEE Transactions on Systems, Man, and Cybernetics, Part B: Cybernetics, vol. 39, no. 6, pp. 1393-1407, Dec 2009.

[32] E. Koutsoupias and C. Papadimitriou, "Worst-case equilibria," in Proc. of the 16th annual conference on Theoretical aspects of computer science. Springer-Verlag, 1999, pp. 404-413.

[33] A. Cournot, Recherches sur les principes mathématiques de la théorie des richesses. L. Hachette, 1838.

[34] H. Tembine, Distributed strategic learning for wireless engineers. CRC Press, 2012. 